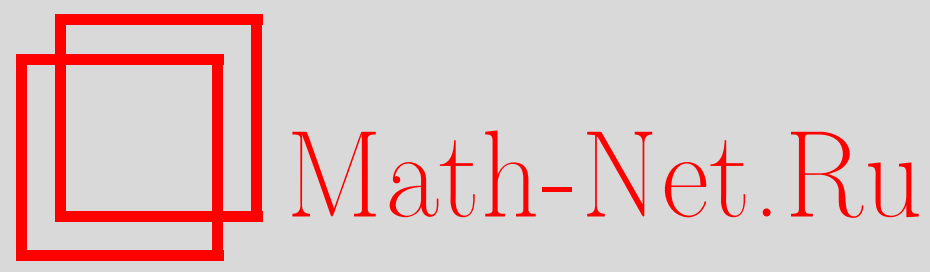

В. Х. Салихов, Г. Г. Вискина, Алгебраические соотношения между гипергеометрической $E$-функцией и ее производными, Матем. заметки, 2002, том 71, выпуск 6, 832-844

DOI: https://doi.org/10.4213/mzm388

Использование Общероссийского математического портала Math-Net.Ru подразумевает, что вы прочитали и согласны с пользовательским соглашением http://www.mathnet.ru/rus/agreement

Параметры загрузки:

IP: 18.209 .158 .208

26 апреля 2023 г., 13:21:29

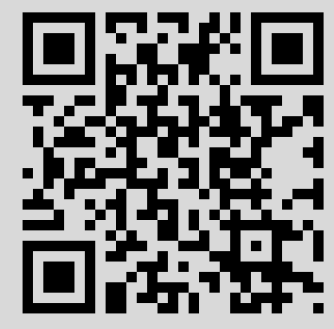




\section{АЛГЕБРАИЧЕСКИЕ СООТНОШЕНИЯ МЕЖДУ ГИПЕРГЕОМЕТРИЧЕСКОЙ Е-ФУНКЦИЕЙ И ЕЕ ПРОИЗВОДНЫМИ}

\section{Г. Г. Вискина, В. Х. Салихов}

В статье рассматривается обобщенная гипергеометрическая функция

$$
\sum_{n=0}^{\infty} \frac{1}{\left(\lambda_{1}+1\right)_{n} \cdots\left(\lambda_{t}+1\right)_{n}}\left(\frac{z}{t}\right)^{t n}, \quad \lambda_{1}, \ldots, \lambda_{t} \in \mathbb{Q} \backslash\{-1,-2, \ldots\},
$$

где $t$ - четное число, и ее производные до порядка $t-1$ включительно. В случае алгебраической зависимости этих функций над $\mathbb{C}(z)$ приводится полная структура алгебраических связей между ними.

Библиография: 8 названий.

Рассмотрим гипергеометрическую $E$-функцию

$$
\varphi(z)=\varphi_{\bar{\lambda}}(z)=\sum_{n=0}^{\infty} \frac{1}{\left(\lambda_{1}+1\right)_{n} \cdots\left(\lambda_{t}+1\right)_{n}}\left(\frac{z}{t}\right)^{t n}
$$

где $\lambda_{i} \in \mathbb{Q},-\lambda_{i} \notin \mathbb{N}, t$ четно, $t=2 \varkappa$.

Функция (1) удовлетворяет дифференциальному уравнению

$$
L_{\bar{\lambda}}(y)=L(y)=\left(\left(\delta+t \lambda_{1}\right) \cdots\left(\delta+t \lambda_{t}\right)-z^{t}\right)(y)=t^{t} \lambda_{1} \cdots \lambda_{t}, \quad \delta=z \frac{d}{d z} .
$$
ций

В работе [1] был установлен критерий алгебраической независимости над $\mathbb{C}(z)$ функ-

$$
\bar{\varphi}=\left(\varphi(z), \delta \varphi(z), \ldots, \delta^{t-1} \varphi(z)\right) .
$$

В настоящей работе описывается полная структура алгебраических связей над $\mathbb{C}(z)$ функций (3) в случае, когда они алгебраически зависимы над $\mathbb{C}(z)$. Полученный результат можно применить для установления оценок снизу мер алгебраической независимости чисел $\varphi(\alpha), \ldots, \delta^{l-1} \varphi(\alpha)$, где $\alpha \in \overline{\mathbb{Q}} \backslash\{0\}, l$ - степень трансцендентности функций $(3)$ над $\mathbb{C}(z)$. Мы ниже остановимся на другом приложении. В работе $[2$, теорема 1 , следствие 1] было установлено эффективное достаточное условие линейной независимости значений $E$-функций над $\overline{\mathbb{Q}}$. С помощью этого результата ниже доказывается следующая

Работа выполнена при частичной поддержке объединенного проекта фонда INTAS и Pоссийского фонда фундаментальных исследований, грант IR-97-1904. 
Tеорема. Пусть $\alpha \in \overline{\mathbb{Q}} \backslash\{0\}$. Тогда иисла $1, \varphi(\alpha), \delta \varphi(\alpha), \ldots, \delta^{t-1} \varphi(\alpha)$ линейно независимы над $\overline{\mathbb{Q}}$.

Отметим, что ввиду критерия, полученного в работе [3], этот результат справедлив и в случае, когда для функции (1) $t$ нечетно. Утверждение теоремы очевидно вьполнено, если функции (3) алгебраически независимы над $\mathbb{C}(z)$.

Всюду далее считаем функции (3) алгебраически зависимыми над $\mathbb{C}(z)$. В этом случае (см. [1]) среди параметров $\lambda_{1}, \ldots, \lambda_{t}$ функции (1) найдется (неотрицательный) целый параметр $\lambda_{i}$. Ввиду возможности провести целочисленные сдвиги $\lambda_{j} \rightarrow \lambda_{j}+m_{j}$, $m_{j} \in \mathbb{Z}$ (подробности см. ниже), будем считать, что этот параметр $\lambda_{i}=0$.

Рассмотрим ряд случаев.

1) Уравнение $L(y)=0$ (см. (2)) линейно неприводимо. В работе [1] показано, что уравнение $L(y)=0$ квадратично приводимо, существуют $X_{0}, \ldots, X_{\varkappa-1} \in \mathbb{Q}$ такие, что $-1 / 2<X_{0}<1 / 2,0 \leqslant X_{i} \leqslant 1 / 2, i=1,2, \ldots, \varkappa-1 ; \varkappa>1$,

$$
\bar{\lambda}=\left(\lambda_{1}, \ldots, \lambda_{t}\right)=X_{0}+\left(0, \frac{1}{2}, X_{1},-X_{1}, \ldots, X_{\varkappa-1},-X_{\varkappa-1}\right) .
$$

Если $X_{0}=0$, то соответствующий единственный многочлен $P$, связьвающий функции (3), построен в [4, с. 469-470]. Напомним конструкцию этого многочлена. Определим числа $\xi_{0}, \ldots, \xi_{t-1} \in \mathbb{Q}$ из равенства

$$
\left(\delta-t \lambda_{1}\right) \cdots\left(\delta-t \lambda_{t}\right)=\delta^{t}+\cdots+\xi_{1} \delta+\xi_{0},
$$

или, учитьвая (4) при $X_{0}=0$,

$$
\delta(\delta-\varkappa)\left(\delta^{2}-t^{2} X_{1}^{2}\right) \cdots\left(\delta^{2}-t^{2} X_{\varkappa-1}^{2}\right)=\delta^{t}+\cdots+\xi_{1} \delta,
$$

где $\xi_{1}=(-1)^{\varkappa} \varkappa t^{2 \varkappa-2} X_{1}^{2} \cdots X_{\varkappa-1}^{2}$.

Далее, определим коэффициенты $\alpha_{r, i} \in \mathbb{Q}$, полагая $\alpha_{r, i}=0$ при $r+i>t+1$; из равенства

$$
\begin{aligned}
& (\delta+\varkappa)\left(\delta^{t-1}+\xi_{t-1} \delta^{t-2}+\cdots+\xi_{1}\right)-\delta(\delta+\varkappa)\left(\delta^{2}-t^{2} X_{1}^{2}\right) \cdots\left(\delta^{2}-t^{2} X_{\varkappa-1}^{2}\right) \\
& \quad=\alpha_{1, t-1} \delta^{t-1}+\cdots+\alpha_{1,0}
\end{aligned}
$$

получим $\alpha_{1, i}=(-1)^{i} \varkappa \xi_{i+1}, i=0,1, \ldots, t-1$; далее,

$$
\begin{aligned}
& (-1)^{r-1}(\delta+\varkappa)\left(\delta^{t-r}+\xi_{t-1} \delta^{t-r-1}+\cdots+\xi_{r}\right) \\
& \quad=\alpha_{r, 0}+\alpha_{r, 1} \delta+\cdots+\alpha_{r, t-r+1} \delta^{t-r+1}, \quad r=2, \ldots, t ;
\end{aligned}
$$

в частности, $\alpha_{r, t-r+1}=(-1)^{r-1}$. Тогда многочлен

$$
P=\sum_{r=1}^{t} \sum_{i=0}^{t-1} \alpha_{r i} z_{r-1} z_{i}+z^{t} z_{0}^{2} \in \mathbb{Q}\left[z^{t}\right]\left[z_{0}, \ldots, z_{t-1}\right] .
$$

дает искомую алгебраическую связь между функциями (3):

$$
P\left(\varphi(z), \ldots, \delta^{t-1} \varphi(z)\right)=C,
$$

где $C=\alpha_{1,0}=\varkappa \xi_{1}=(1 / 4)(-1)^{\varkappa} t^{t} X_{1}^{2} \cdots X_{\varkappa-1}^{2}$.

Многочлен $P$ неприводим над $\overline{\mathbb{C}(z)}$, так как уравнение $L(y)=0$ в рассматриваемом случае линейно неприводимо над $\overline{\mathbb{C}(z)}$. Для доказательства теоремы понадобится еще одно свойство многочлена $P$. 
Лемма 1. Для всех $\theta \in \mathbb{C}$ многочлен

$$
P_{\theta}=\sum_{r=1}^{t} \sum_{i=0}^{t-1} \alpha_{r i} z_{r-1} z_{i}+\theta^{t} z_{0}^{2}
$$

неприводим в $\mathbb{C}\left[z_{0}, \ldots, z_{t-1}\right]$.

ДокАЗАТЕЛЬСТво. Предположим противное. Тогда

$$
P_{\theta}=\left(a_{0} z_{0}+\cdots+a_{t-1} z_{t-1}\right)\left(b_{0} z_{0}+\cdots+b_{t-1} z_{t-1}\right)
$$

где все $a_{i}, b_{i} \in \mathbb{C}, a_{t-1} \neq 0$.

Определим $s$ из условий $b_{s} \neq 0, b_{i}=0$ при $i>s$. Так как $\alpha_{r, i}=0$ при $r+i>t+1$, то, сравнивая коэффициенты при $z_{t-1} z_{s}$ в обеих частях равенства (8), получим $s \leqslant 1$. $\mathrm{C}$ другой стороны, коэффициент при $z_{t-2} z_{2}$ в многочлене $P_{\theta}$ равен $\alpha_{t-1,2}+\alpha_{3, t-2}=$ $1+1=2$, если $t>4$, или $\alpha_{3,2}=1$, если $t=4$. Так как в правой части (8) это слагаемое отсутствует, то (8) невозможно, и лемма доказана.

Переход к случаю $X_{0} \neq 0$ проведем, как в работе [4, с. 463]. Пусть $L_{0}$ - оператор $L$ из (2), отвечающий $X_{0}=0$ в случае (4). Отображение $v=s\left(t X_{0}\right) u$ осуществляет изоморфизм пространств решений уравнений $L_{0}(v)=0$ и $L(u)=0$. Здесь и далее используется введенное в [5] обозначение $s(\alpha), \alpha \in \mathbb{C}$, для фиксированного нетривиального решения уравнения $y^{\prime}=(\alpha / z) y$; всюду $\alpha \in \mathbb{Q}$, поэтому можно считать, что $s(\alpha)=z^{\alpha}$ - некоторая фиксированная ветвь показательной функции. Пусть $\left(x+t X_{0}\right)^{r}=x^{r}+c_{r, r-1} x^{r-1}+\cdots+c_{r, 0}, r=1, \ldots, t-1$,

$$
\bar{P}=P\left(z_{0}, z_{1}+c_{1,0} z_{0}, \ldots, z_{t-1}+c_{t-1, t-2} z_{t-2}+\cdots+c_{t-1,0} z_{0}\right) .
$$

Для любого решения $v$ уравнения $L_{0}(v)=0$ имеем аналогично $(6) P\left(v, \ldots, \delta^{t-1} v\right)=$ $c \in \mathbb{C}$. Поэтому из (9) получаем

$$
c=P\left(v, \ldots, \delta^{t-1} v\right)=P\left(s\left(t X_{0}\right) u, \ldots, \delta^{t-1}\left(s\left(t X_{0}\right) u\right)\right)=s\left(2 t X_{0}\right) \bar{P}\left(u, \ldots, \delta^{t-1} u\right),
$$

или

$$
\bar{P}\left(u, \ldots, \delta^{t-1} u\right)=c(u) s\left(-2 t X_{0}\right)
$$

где $u$ - произвольное решение уравнения $L(u)=0, c(u) \in \mathbb{C}$.

В частности (см. (2) и условие $\left.\lambda_{i}=0\right)$,

$$
\bar{P}\left(\varphi(z), \ldots, \delta^{t-1} \varphi(z)\right)=c \cdot s\left(-2 t X_{0}\right), \quad c \in \mathbb{Q} .
$$

Так как левая часть равенства (10) имеет вид $\sum_{n=0}^{\infty} c_{n} z^{t n}, c_{n} \in \mathbb{Q}($ см. (1), (5) и (9)), то ввиду оценки $-t<\left(-2 t X_{0}\right)<t, X_{0} \neq 0$, получаем $c=0$ в $(10)$, т.е.

$$
\bar{P}\left(\varphi(z), \ldots, \delta^{t-1} \varphi(z)\right)=0
$$


Рассмотрим теперь влияние целочисленных сдвигов параметров $\lambda_{j}$ на соотношения $(6),(11)$. Пусть $\psi(z)$ - функция вида $(1)$ с $\lambda_{1} \rightarrow\left(\lambda_{1}-1\right), \lambda_{1} \neq 0$. Тогда (см. [5, с. 254])

$$
\left(\begin{array}{c}
\psi \\
\delta \psi \\
\vdots \\
\delta^{t-1} \psi
\end{array}\right)=\left(\begin{array}{cccccc}
1 & \frac{1}{t \lambda_{1}} & 0 & 0 & \ldots & 0 \\
0 & 1 & \frac{1}{t \lambda_{1}} & 0 & \ldots & 0 \\
\ldots \ldots \ldots \ldots \ldots & \ldots & \ldots & \ldots & \ldots \\
0 & 0 & \ldots & 0 & 1 & \frac{1}{t \lambda_{1}} \\
\frac{z^{t}}{t \lambda_{1}}+c_{0} & c_{1} & c_{2} & c_{3} & \ldots & c_{t-1}
\end{array}\right)\left(\begin{array}{c}
\varphi \\
\delta \varphi \\
\vdots \\
\delta^{t-1} \varphi
\end{array}\right)+\left(\begin{array}{c}
0 \\
\vdots \\
0 \\
c
\end{array}\right)
$$

где $c, c_{i} \in \mathbb{Q}$; определитель вьписанной матрицы линейного невырожденного преобразования равен $(-1)^{t+1} z^{t} /\left(t \lambda_{1}\right)^{t}$.

Следовательно, изменение нескольких параметров $\lambda_{j}$ вида $\lambda_{j} \rightarrow \lambda_{j}+m_{j}, m_{j} \in \mathbb{Z}$, приводят к преобразованию вида

$$
\left(\psi, \ldots, \delta^{t-1} \psi\right)=\left(\varphi, \ldots, \delta^{t-1} \varphi\right) \cdot A+\left(a_{0}, \ldots, a_{t-1}\right),
$$

где $A=\left(a_{i j}\right)_{i, j=1, \ldots, t}, a_{i j}=\sum_{r=-M}^{N} c_{i j r} z^{r t}, c_{i j r} \in \mathbb{Q}, \operatorname{det} A=c \cdot z^{d t}, c \in \mathbb{Q} \backslash\{0\}$, $d=N-M, a_{k}=\sum_{r=-M}^{N} c_{k r} z^{r t}, c_{k r} \in \mathbb{Q}, N-$ количество сдвигов параметров типа $\lambda_{j} \rightarrow \lambda_{j}-1, M-$ количество сдвигов параметров типа $\lambda_{j} \rightarrow \lambda_{j}+1$.

Подстановка $\left(z_{0}, \ldots, z_{t-1}\right) \rightarrow\left(z_{0}, \ldots, z_{t-1}\right) A+\left(a_{0}, \ldots, a_{t-1}\right)$ в многочлен $P$ в слу-

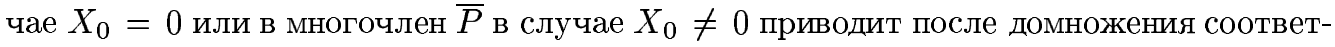
ственно (6) или (11) на некоторьй моном $z^{s t}, s \in \mathbb{Z}$, к неприводимому квадратичному соотношению между функциями (3) с коэффищиентами из $\mathbb{Q}\left[z^{t}\right]$.

2) Уравнение $L(y)=0$ линейно приводимо. В этом случае (см. [5]) существует делитель $g$ числа $t$ такой, что

$$
\bar{\lambda}+\frac{1}{g} \sim \bar{\lambda} .
$$

Будем считать $g$ максимально возможным.

2.1) $g=t$. Так как существует $\lambda_{i} \in \mathbb{Z}^{+}$, то из (13) находим

$$
\bar{\lambda} \sim\left(0,-\frac{1}{t}, \ldots,-\frac{t-1}{t}\right) .
$$

Мы ограничимся рассмотрением ситуации, когда

$$
\bar{\lambda}=\left(0,-\frac{1}{t}, \ldots,-\frac{t-1}{t}\right) .
$$

Соответствующие целочисленные сдвиги можно провести, как и в случае 1). Как в работе [5, равенство (114)], получаем

$$
\varphi(z)=\frac{1}{t} \sum_{\nu=0}^{t-1}\left(\exp \xi^{\nu} z\right)
$$


здесь и далее $\xi-$ примитивньй корень степени $t$ из 1. Имеем

$$
\left(\varphi(z), \ldots, \varphi^{(t-1)}(z)\right)=\frac{1}{t}\left(e^{z}, e^{\xi z}, \ldots, e^{\xi^{t-1} z}\right) \cdot C, \quad \text { где } C=\left(\xi^{(i-1)(j-1)}\right)_{i, j=1, \ldots, t} .
$$

Алгебраические связи между функциями $\varphi(z), \ldots, \varphi^{(t-1)}(z)$ над $\mathbb{C}(z)$ ввиду $(14)$ сводятся к алгебраическим связям между функциями $e^{z}, e^{\xi z}, \ldots, e^{\xi^{t-1} z}$. Пусть $m-$ значение функции Эйлера от $t$, а $x^{m}-n_{m-1} x^{m-1}-\cdots-n_{0}-$ примитивный многочлен над $\mathbb{Q}$ числа $\xi$. Тогда

$$
\exp \left(\xi^{m} z\right)=\prod_{\nu=0}^{m-1}\left(\exp \left(\xi^{\nu} z\right)\right)^{n_{\nu}}, \quad n_{\nu} \in \mathbb{Z} .
$$

Аналогичные выражения всех $\exp \left(\xi^{k} z\right), k=m+1, \ldots, t-1$, через базис трансцендентности над $\mathbb{C}(z)$ - набор экспонент $\exp \left(\xi^{\nu} z\right), \nu=0, \ldots, m-1,-$ получаются тривиально.

2.2) $\tau=t / g$ четно. В этом случае $\left[1\right.$, с. 283] существуют $X_{0}^{\prime}, \ldots, X_{s}^{\prime} \in \mathbb{Q}, s=\tau / 2-1$, такие, что

$$
\begin{gathered}
-\frac{1}{2}<X_{0}^{\prime}<\frac{1}{2}, \quad 0 \leqslant X_{i}^{\prime} \leqslant \frac{1}{2}, \quad i=1, \ldots, s, \quad s \geqslant 1, \\
\bar{\lambda} \sim\left(\frac{\gamma_{j}-k}{g}, j=1, \ldots, \tau, k=0,1, \ldots, g-1\right)
\end{gathered}
$$

где

$$
\bar{\gamma}=\left(\gamma_{1}, \ldots, \gamma_{\tau}\right)=X_{0}^{\prime}+\left(0, \frac{1}{2} ; X_{1}^{\prime},-X_{1}^{\prime} ; \ldots ; X_{s}^{\prime},-X_{s}^{\prime}\right),
$$

причем $\gamma_{j}=0$ для некоторого $j$. Учитьвая возможность провести целочисленные сдвиги параметров $\lambda_{j}$, как и в $[3$, равенство $(60)]$, можно считать, что

$$
\varphi_{\bar{\lambda}}(z)=\frac{1}{g} \sum_{\nu=0}^{g-1} \varphi_{\bar{\gamma}}\left(\xi^{\nu} z\right)
$$

Пусть $\mathscr{F}=\left(f_{1}(z), \ldots, f_{t}(z)\right), G=\left(g_{1}(z), \ldots, g_{t}(z)\right)$, где все $f_{i}(z), g_{i}(z)-$ аналитические функции. Будет удобно ввести отношение эквивалентности $\mathscr{F} \sim G$, если $G=\mathscr{F} \cdot \mathscr{D}+\mathscr{D}_{0}$, где $\mathscr{D}=\left(d_{i j}\right)_{i, j=1, \ldots, t}, \mathscr{D}_{0}=\left(d_{0,1}, \ldots, d_{0, t}\right)$, все $d_{i j}=\sum_{k=k_{1}}^{k_{2}} d_{i j k} z^{k}$, все $d_{i j k} \in \overline{\mathbb{Q}} ; k_{1}, k_{2} \in \mathbb{Z} ; \operatorname{det} \mathscr{D}=c z^{s}, c \in \overline{\mathbb{Q}} \backslash\{0\}, s \in \mathbb{Z}$.

ЗАмЕчание 1. Вьше было показано, что целочисленные сдвиги параметров у функции (1) преобразуют набор (3) в эквивалентньй набор функций. Кроме того, очевидно, что теорему достаточно доказать для любого набора $E$-функций эквивалентного набоpy (3).

В условиях (16) в работе [3, с. 208-209] было показано, что

$$
\bar{\varphi} \sim\left(\delta^{i} \varphi \bar{\gamma}\left(\xi^{\nu} z\right), i=0, \ldots, \tau-1, \nu=0, \ldots, g-1\right) .
$$

Функция $\varphi_{\bar{\gamma}}\left(\xi^{\nu} z\right)$ является решением уравнения

$$
L_{\nu}(y)=\left(\left(\delta+\tau \gamma_{1}\right) \cdots\left(\delta+\tau \gamma_{\tau}\right)-\xi^{\nu \tau} z^{\tau}\right)(y)=0 .
$$

Для функции $\varphi \bar{\gamma}\left(\xi^{\nu} z\right)$ выполнены все условия случая 1) (см. (15)): существует $\gamma_{i}=0$, уравнение (18) линейно неприводимо ввиду выбора в (13) максимального $g$. Поэтому 
после замены $t \rightarrow \tau, \bar{\lambda} \rightarrow \bar{\gamma}, z \rightarrow \xi^{\nu} z,\left(z_{0}, \ldots, z_{t-1}\right) \rightarrow \bar{z}_{\nu}=\left(z_{0, \nu}, \ldots, z_{\tau-1, \nu}\right)$ в многочлене (5) для каждого набора функций

$$
F_{\nu}=\left\{\varphi \bar{\gamma}\left(\xi^{\nu} z\right), \ldots, \delta^{(\tau-1)} \varphi_{\bar{\gamma}}\left(\xi^{\nu} z\right)\right\}
$$

получаем соотношение вида (6) при $X_{0}^{\prime}=0$ либо вида (11) при $X_{0}^{\prime} \neq 0$.

Обозначим полученные многочлены от переменных $\bar{z}_{\nu}$ соответственно через $P_{\nu}$ и $\bar{P}_{\nu}$. Пусть

$$
\begin{aligned}
\bar{z}=\left(\bar{z}_{0}, \ldots, \bar{z}_{g-1}\right) & =\left(z_{i, v}, i=0, \ldots, \tau-1, \nu=0, \ldots, g-1\right), \\
C & =\frac{1}{4}(-1)^{s+1} \tau^{\tau} X_{1}^{\prime 2} \cdots X_{s}^{\prime 2}
\end{aligned}
$$

ЛЕмма 2. Идеал $J$, порожсденный в кольие $\mathbb{C}(z)[\bar{z}]$ набором $g$ многочленов

$$
\left\{P_{0}\left(\bar{z}_{0}\right)-C, \ldots, P_{g-1}\left(\bar{z}_{g-1}\right)-C\right\}
$$

либо набором $g$ многочленов

$$
\left\{\bar{P}_{0}\left(\bar{z}_{0}\right), \ldots, \bar{P}_{g-1}\left(\bar{z}_{g-1}\right)\right\}
$$

является идеалом всех алгебраических связей над $\mathbb{C}(z)$ междуу функииями (17) соответственно в случае $X_{0}^{\prime}=0$ и в случае $X_{0}^{\prime} \neq 0$.

ДокАЗАТЕЛЬСтво. Осуществим для каждой функции $\varphi \bar{\gamma}\left(\xi^{\nu} z\right)$ замену переменной $(z / \tau)^{\tau} \rightarrow z$ и обозначим полученную функцию через $f_{\nu}(z)$. Функция $f_{\nu}(z)$ является решением уравнения (см. (18))

$$
\bar{L}_{\nu}(y)=\left(\left(\delta+\gamma_{1}\right) \cdots\left(\delta+\gamma_{\tau}\right)-\xi^{\nu \tau} z\right)(y)=0
$$

Обозначим через $\Phi_{\nu}$ расширение Пикара-Вессио поля $\mathbb{C}(z)$ для уравнения $(20)$, содержащее функцию $f_{\nu}(z)$ (соответствующая фундаментальная система решений подробно описана, например, в [5, с. 235]). Пусть $\Phi$ - аналогичное расширение ПикараВессио для совокупности уравнений $(20)$ при $\nu=0, \ldots, g-1 ; G_{\nu}, G$ - соответствующие этим расширениям дифференциальные группы Галуа; $G_{\nu}^{0, \mathrm{der}}, G^{0, \mathrm{der}}$ - соответствующие коммутаторные группы для связных компонент единиц $G_{\nu}^{0}, G^{0}$ групा $G_{\nu}, G$.

Согласно $[6$, предложение 3.8 .2$]$ с учетом того, что уравнения $(20)$ при $\nu=0, \ldots, g-1$ попарно некогредиентны и неконтргредиентны (см. [1, с. 283] и [7, с. 300]) получаем

$$
G^{0, \mathrm{der}}=\prod_{\nu=0}^{g-1} G_{\nu}^{0, \mathrm{der}}
$$

По следствию 3.6.1 и теореме 3.6 работы $[6]$ все $G_{\nu}^{0, \operatorname{der}}=G_{\nu}^{0}=S p(\tau), \nu=0, \ldots, g-1$. Из (21) находим (см. также [8, с. 83])

$$
\operatorname{dim} G \geqslant \operatorname{dim} G^{0, \operatorname{der}}=\sum_{\nu=0}^{g-1} \operatorname{dim} G_{\nu}^{0, \operatorname{der}}=\sum_{\nu=0}^{g-1} \operatorname{dim} G_{\nu}^{0}=\sum_{\nu=0}^{g-1} \operatorname{dim} G
$$


С другой стороны, очевидно, что

$$
\operatorname{dim} G \leqslant \sum_{\nu=0}^{g-1} \operatorname{dim} G_{\nu}
$$

Поэтому

$$
\operatorname{dim} G=\sum_{\nu=0}^{g-1} \operatorname{dim} G_{\nu}
$$

В частности, база трансцендентности поля $\Phi$ над $\mathbb{C}(z)$ может быть получена объединением баз трансцендентности всех полей $\Phi_{0}, \ldots, \Phi_{g-1}$ над $\mathbb{C}(z)$. Так как, очевидно (см. (19)), что

$$
\tau-1=\operatorname{deg} \operatorname{tr}_{\mathbb{C}(z)} F_{\nu}=\operatorname{deg} \operatorname{tr}_{\mathbb{C}(z)}\left(f_{\nu}(z), \ldots, \delta^{\tau-2} f_{\nu}(z)\right),
$$

то $(\tau-1) g=\operatorname{deg} \operatorname{tr}_{\mathbb{C}(z)}\left(\delta^{i} f_{\nu}(z), i=0, \ldots, \tau-2, \nu=0, \ldots, g-1\right)$. Поэтому степень трансцендентности над $\mathbb{C}(z)$ набора функций $(17)$ также равна $(\tau-1) g=t-g$. Ввиду неприводимости многочленов $P_{\nu}\left(\bar{z}_{\nu}\right)-C$ (соответственно $\left.\bar{P}_{\nu}\left(\bar{z}_{\nu}\right)\right)$ в кольце $\overline{\mathbb{C}(z)}\left[\bar{z}_{\nu}\right]$ главный идеал $\left(P_{\nu}-C\right)$ (соответственно $\left.\left(\bar{P}_{\nu}\right)\right)$ определяет многообразие, и поэтому [8, c. 80] идеал $J$ простой с общей точкой (17). Лемма доказана.

2.3) $\tau=t / g$ нечетно, $\tau>1$. В этом случае (см. [1, с. 283-284]) вместо (15) вьполнено соотношение

$$
\bar{\gamma}=\left(\gamma_{1}, \ldots, \gamma_{\tau}\right)=X_{0}^{\prime}+\left(0, X_{1}^{\prime},-X_{1}^{\prime}, \ldots, X_{s}^{\prime},-X_{s}^{\prime}\right), \quad s=\frac{\tau-1}{2} .
$$

Пусть $G$ - дифференциальная группа Галуа для совокупности уравнений (20) при $\nu=0, \ldots, g / 2-1$. Сохраним в остальном все обозначения, введенные при рассмотрении случая 2.2). Аналогично получим, что уравнения (20) при $\nu=0, \ldots, g / 2-1$ попарно некогредиентны и неконтргредиентны; $g / 2$ пар уравнений

$$
\bar{L}_{\nu}(y)=0, \quad \bar{L}_{\nu+g / 2}(y)=0, \quad \nu \in\left\{0, \ldots, \frac{g}{2}-1\right\}
$$

контргредиентны. Вместо (21) получим по предложению 3.8.2 из [6] представление

$$
G^{0, \mathrm{der}}=\prod_{\nu=0}^{g / 2-1} G_{\nu}^{0, \mathrm{der}}
$$

По теореме 3.6 работы [6] в рассматриваемом случае все $G_{\nu}^{0, \text { der }}=G_{\nu}^{0}=S L(\tau)$, значит, как в случае 2.2 ), из (23) получаем

$$
\operatorname{dim} G=\sum_{\nu=0}^{g / 2-1} \operatorname{dim} G_{\nu}
$$

Кроме того, нам понадобится соотношение

$$
\operatorname{dim} G_{\nu}=\operatorname{dim} G_{\nu}^{0}=\operatorname{dim} S L(\tau)=\tau^{2}-1 .
$$


Обозначим через $U_{\nu} \subset \Phi_{\nu}$ линейное пространство всех решений уравнения (20), через $\bar{L}_{\nu}^{*}$ сопряженный оператор для оператора $\bar{L}_{\nu}, \nu=0, \ldots, g / 2-1$.

Пусть $\nu \in\{0, \ldots, g / 2-1\}, u \in U_{\nu}, v \in U_{\nu+g / 2}, \bar{v}=s\left(2 X_{0}-1\right) v$. Положим $u_{0}=u$, $u_{k}=\left(\delta+\gamma_{1}\right) \cdots\left(\delta+\gamma_{k}\right) u, k=1, \ldots, \tau-1 ; \bar{v}_{0}=\bar{v}, \bar{v}_{k}=\left(\delta+1-\gamma_{\tau-k+1}\right) \cdots\left(\delta+1-\gamma_{\tau}\right) \bar{v}$, $k=1, \ldots, \tau-1$.

Интегрируя $\tau$ раз по частям, находим

$$
\begin{aligned}
\int \bar{L}_{\nu}(u) \bar{v} d z & =\int\left(\delta+\gamma_{\tau}\right) u_{\tau-1} \bar{v} d z-\int z \xi^{\nu \tau} u \bar{v} d z \\
& =z u_{\tau-1} \bar{v}-\int u_{\tau-1} \bar{v}_{1} d z+\int z \xi^{(\nu+g / 2) \tau} u \bar{v} d z=\cdots \\
& =z \sum_{j=0}^{\tau-1}(-1)^{j} u_{j} \bar{v}_{\tau-j-1}-\int \bar{L}_{\nu}^{*}(\bar{v}) u d z
\end{aligned}
$$

где $\bar{L}_{\nu}^{*}(\bar{v})=\left(\delta+1-\gamma_{1}\right) \cdots\left(\delta+1-\gamma_{\tau}\right)-\xi^{(\nu+g / 2) \tau} \cdot z$.

Из $(22)$ имеем $1-\bar{\gamma}=1-2 X_{0}^{\prime}+\bar{\gamma}^{\prime}$, где $\gamma_{1}^{\prime}=\gamma_{1}, \gamma_{2 k}^{\prime}=\gamma_{2 k+1}, \gamma_{2 k+1}^{\prime}=\gamma_{2 k}, k=1, \ldots, s$.

Следовательно, $\bar{L}_{\nu}^{*}(\bar{v})=s\left(2 X_{0}^{\prime}-1\right) \cdot \bar{L}_{\nu+g / 2}(v)=0$; значит, из $(26)$ ввиду $\bar{L}_{\nu}(u)=0$ получаем

$$
z \cdot \sum_{j=0}^{\tau-1}(-1)^{j} u_{j} \bar{v}_{\tau-j-1}=c(u, v) \in \mathbb{C}
$$

или в обозначениях $v_{0}=v, v_{k}=\left(\delta+\gamma_{\tau-k+1}^{\prime}\right) \cdots\left(\delta+\gamma_{\tau}^{\prime}\right) v, k=1, \ldots, \tau-1$, с учетом $\left(\delta+1-\gamma_{j}\right)\left(s\left(2 X_{0}^{\prime}-1\right) v\right)=s\left(2 X_{0}^{\prime}-1\right)\left(\delta+\gamma_{j}^{\prime}\right) v$ заключаем

$$
s\left(2 X_{0}^{\prime}\right) \cdot \sum_{j=0}^{\tau-1}(-1)^{j} u_{j} v_{\tau-j-1}=c(u, v) .
$$

В частности, соотношение (27) справедливо для $u=f_{\nu}(z), v=f_{\nu+g / 2}(z)$. Далее покажем, что соотношения (27) являются искомыми базисньми соотношениями. Для этого нам понадобится несколько вспомогательных утверждений.

Лемма 3. Пусть $m>2$, функиии $a_{i j} \in \Phi_{\nu}, i=1, \ldots, m-1, j=1, \ldots, m$, алгебраически независимы над $\mathbb{C}(z), \Delta_{k}=\operatorname{det}\left(a_{i j}\right)_{i=1, \ldots, m-1, j=1, \ldots, m, j \neq k}$. Тогда функции $a_{1,1}, \ldots, a_{1, m-1}, \Delta_{1}, \ldots, \Delta_{m}$ алгебраически независимы над $\mathbb{C}(z)$.

ДокАЗАТЕЛьСтво. Докажем лемму индукцией по $m$. При $m=3$ для доказательства леммы достаточно установить следующее: если

$$
F_{1}=\mathbb{C}(z)\left(a_{1}, a_{2}, a_{3}, b_{1}, b_{2}, b_{3}\right), \quad \operatorname{deg} \operatorname{tr}_{\mathbb{C}(z)} F_{1}=6
$$

то

$$
\left\{a_{1}, a_{2}, b_{1}, \Delta_{1}=\left|\begin{array}{cc}
a_{2} & a_{3} \\
b_{2} & b_{3}
\end{array}\right|, \Delta_{2}=\left|\begin{array}{cc}
a_{1} & a_{3} \\
b_{1} & b_{3}
\end{array}\right|, \Delta_{3}=\left|\begin{array}{cc}
a_{1} & a_{2} \\
b_{1} & b_{2}
\end{array}\right|\right\}
$$

- базис трансцендентности поля $F_{1}$ над $\mathbb{C}(z)$. Из очевидных соотношений

$$
a_{1} b_{2}-a_{2} b_{1}=\Delta_{3}, \quad b_{1} \Delta_{1}-b_{2} \Delta_{2}+b_{3} \Delta_{3}=0, \quad a_{1} \Delta_{1}-a_{2} \Delta_{2}+a_{3} \Delta_{3}=0
$$


легко получаем рациональные выражения для $b_{2}, b_{3}, a_{3}$ в терминах $a_{1}, a_{2}, b_{1}, \Delta_{1}, \Delta_{2}, \Delta_{3}$, поэтому утверждение леммы при $m=3$ выполнено.

Проведем шаг индукции $m \rightarrow m+1$. Пусть в условии леммы $m \rightarrow m+1$ и для некоторого $\left\{a_{i j}\right\}$ утверждение леммы не вьполнено, т.е.

$$
\sum_{s_{i} \in \mathbb{Z}^{+}} r_{\bar{s}} a_{11}^{s_{1}} \cdots a_{1 m}^{s_{m}} \Delta_{1}^{s_{m+1}} \cdots \Delta_{m+1}^{s_{2 m+1}}=0
$$

где все $r_{\bar{s}} \in \mathbb{C}(z), r_{\bar{s}} \neq 0$, и суммирование в (28) проходит по некоторому конечному множеству $\bar{s}=\left(s_{1}, \ldots, s_{2 m+1}\right)$.

Вычисляя в (28) коэффициент при старшей степени $a_{m_{1}}^{t}$, получаем

$$
\sum r_{\bar{s}} a_{11}^{s_{1}} \cdots a_{1 m}^{s_{m}} \Delta_{1}^{s_{m+1}} \Delta_{2}^{s_{m+2}} \cdots \Delta_{m+1}^{s_{2 m+1}}=0
$$

где суммирование распространяется на все $\bar{s}$ такие, что $r_{\bar{s}} \neq 0, t=s_{m+2}+\cdots+s_{2 m+1}$ максимально возможное;

$$
\Delta_{k}^{\prime}=\operatorname{det}\left(a_{i j}\right), \quad i=1, \ldots, m-1, \quad j=2, \ldots, m+1, \quad j \neq k, \quad k \in\{2, \ldots, m+1\} .
$$

Раскладьвая $\Delta_{1}$ по последней строке, находим

$$
\Delta_{1}=\omega_{2} a_{m 2}+\cdots+\omega_{m+1} a_{m, m+1}
$$

причем все $\omega_{j} \neq 0, \omega_{i} \in F_{2}$, где $F_{2}=\mathbb{C}(z)\left(a_{i j}, i=1, \ldots, m-1, j=1, \ldots, m+1\right)$.

Так как $a_{m 2}, \ldots, a_{m, m+1}$ алгебраически независимы над $F_{2}$, то $\Delta_{1}$ трансцендентен над $F_{2}$, поэтому из равенства (29) получаем нетривиальное соотношение вида

$$
\sum r_{\bar{s}} a_{11}^{s_{1}} \cdots a_{1 m}^{s_{m}} \Delta_{2}^{s_{m+2}} \cdots \Delta_{m+1}^{s_{2 m+1}}=0
$$

Так как $\Delta_{2}^{\prime}, \ldots, \Delta_{m+1}^{\prime}$ не содержат $a_{11}$, то из последнего соотношения аналогично имеем

$$
\sum r_{\bar{s}} a_{12}^{s_{2}} \cdots a_{1 m}^{s_{m}} \Delta_{2}^{s_{m+2}} \cdots \Delta_{m+1}^{s_{2 m+1}}=0
$$

Равенство (30) противоречит индуктивному предположению для множества $\left\{a_{i j}\right.$, $i=1, \ldots, m-1, j=2, \ldots, m+1\}$, и лемма доказана.

ЛЕмма 4. Пусть $\varphi_{0}, \ldots, \varphi_{\tau-1}-$ произвольный базис $U_{\nu}$. Тогда функиии

$$
\varphi_{0}, \delta \varphi_{0}, \ldots, \delta^{\tau-2} \varphi_{0}, \delta^{j} \varphi_{i}, \quad i=0, \ldots, \tau-1, \quad j=1, \ldots, \tau-1
$$

алгебраически независимы над $\mathbb{C}(z)$. 
ДОКАЗАТЕЛЬСТВО. Пусть $y_{1}, \ldots, y_{r} \in U_{\nu}$.

Пусть $W\left(y_{1}, \ldots, y_{r}\right)=\operatorname{det}\left(\delta^{j-1} y_{i}\right)_{i, j=1, \ldots, r}-$ вронскиан. Из (22) и уравнения (20) имеем

$$
\delta W\left(\varphi_{0}, \ldots, \varphi_{\tau-1}\right)=-\tau X_{0}^{\prime} \cdot W\left(\varphi_{0}, \ldots, \varphi_{\tau-1}\right),
$$

т.е.

$$
W\left(\varphi_{0}, \ldots, \varphi_{\tau-1}\right)=c \cdot s\left(-\tau X_{0}^{\prime}\right), \quad c \in \mathbb{C} \backslash\{0\} .
$$

Ввиду (25) выполнено $\operatorname{deg} \operatorname{tr}_{\mathbb{C}(z)}\left(\delta^{j} \varphi_{i}, i, j=0, \ldots, \tau-1\right)=\tau^{2}-1$.

$\mathrm{C}$ другой стороны, раскладывая $W\left(\varphi_{0}, \ldots, \varphi_{\tau-1}\right)$ по первой строке, находим

$$
\varphi_{0} W_{0}+\cdots+\delta^{\tau-1} \varphi_{0} W_{\tau-1}=c \cdot s\left(-\tau X_{0}^{\prime}\right),
$$

где все

$$
\begin{gathered}
W_{k} \in F_{1}=\mathbb{C}(z)\left(\delta^{j} \varphi_{i}, i=1, \ldots, \tau-1, j=0, \ldots, \tau-1\right), \\
W_{\tau-1}=W\left(\varphi_{1}, \ldots, \varphi_{\tau-1}\right) \neq 0 .
\end{gathered}
$$

Следовательно, $\delta^{\tau-1} \varphi_{0}$ алгебраичен над $F_{1}\left(\varphi_{0}, \ldots, \delta^{\tau-2} \varphi_{0}\right)$,

$$
\tau^{2}-1=\operatorname{deg} \operatorname{tr}_{\mathbb{C}(z)}\left(\delta^{j} \varphi_{i}, i, j=0, \ldots, \tau-1,(i, j) \neq(0, \tau-1)\right),
$$

и лемма доказана.

Лемма 5. Пусть $\nu \in\{0, \ldots, g / 2-1\}, u \in U_{\nu}, v \in U_{\nu+g / 2}, u \neq 0, v \neq 0$. Тогда $\operatorname{deg} \operatorname{tr}_{\mathbb{C}(z)}\left(u, \delta u, \ldots, \delta^{\tau-1} u, v, \delta v, \ldots, \delta^{\tau-1} v\right)=2 \tau-1$.

ДоказАТеЛЬСтво. Необходимо доказать, что (27) - единственное соотношение над $\overline{\mathbb{C}(z)}$ меж ду рассматриваемыми элементами. Рассмотрим два случая: а) $c(u, v) \neq 0$; б) $c(u, v)=0$.

a) Фиксируем $\left\{\psi_{0}=u / c(u, v), \psi_{1}, \ldots, \psi_{\tau-1}\right\}$ - базис $U_{\nu}$ такой, что $c\left(\psi_{0}, v\right)=1$, $c\left(\psi_{i}, v\right)=0, i=1, \ldots, \tau-1, W\left(\psi_{0}, \ldots, \psi_{\tau-1}\right)=s\left(-\tau X_{0}^{\prime}\right)$. Такой базис существует ввиду линейности $c(u, v)$ по $u$ (см. также доказательство леммы 4$)$.

Положим $a_{i j}=(-1)^{j}\left(\delta+\gamma_{1}\right) \cdots\left(\delta+\gamma_{j}\right) \psi_{i}, i, j=0, \ldots, \tau-1$. Из равенств $(27)$ при $u=\psi_{i}, i=0, \ldots, \tau-1$, получаем

$$
v=v_{0}=\frac{\operatorname{det} B}{s\left(2 X_{0}^{\prime}\right) \operatorname{det} A},
$$

где $A=\left(a_{i j}\right)_{i, j=0, \ldots, \tau-1}, B=\left(a_{i j}\right)_{i=1, \ldots, \tau-1, j=0, \ldots, \tau-2}$, т.е.

$\operatorname{det} A=(-1)^{\tau(\tau-1) / 2} W\left(\psi_{0}, \ldots, \psi_{\tau-1}\right), \quad \operatorname{det} B=(-1)^{(\tau-1)(\tau-2) / 2} W\left(\psi_{1}, \ldots, \psi_{\tau-1}\right)$,

и поэтому

$$
v=s\left((\tau-2) X_{0}^{\prime}\right) \widetilde{v}, \quad \widetilde{v}=W\left(\psi_{1}, \ldots, \psi_{\tau-1}\right) \in \Phi_{\nu} .
$$

Для доказательства леммы в рассматриваемом случае достаточно показать, что

$$
\operatorname{deg} \operatorname{tr}_{\mathbb{C}(z)}\left(\psi_{0}, \delta \psi_{0}, \ldots, \delta^{\tau-2} \psi_{0}, \widetilde{v}, \delta \widetilde{v}, \ldots, \delta^{\tau-1} \widetilde{v}\right)=2 \tau-1 .
$$

Введем обозначение

$$
\omega_{k}=\operatorname{det}\left(\delta^{j} \psi_{i}\right)_{i=1, \ldots, \tau-1, j=0, \ldots, \tau-1, j \neq \tau-1-k}, \quad k=0, \ldots, \tau-1 ;
$$


при этом из $(31)$ и (33) имеем $\omega_{0}=\widetilde{v}, \omega_{1}=\delta \widetilde{v}$. Дифференцируя $\omega_{k}$ и учитьвая, что $\psi_{1}, \ldots, \psi_{\tau-1} \in U_{\nu}$, получаем соотношение вида

$$
\omega_{i}=\delta^{i} \widetilde{v}+r_{i, i-1} \delta^{i-1} \widetilde{v}+\cdots+r_{i, 0} \widetilde{v}, \quad i=0, \ldots, \tau-1,
$$

где все $r_{i, j} \in \overline{\mathbb{Q}}[z]$. Следовательно, соотношение (32) равносильно условию

$$
\operatorname{deg} \operatorname{tr}_{\mathbb{C}(z)}\left(\psi_{0}, \delta \psi_{0}, \ldots, \delta^{\tau-2} \psi_{0}, \omega_{0}, \ldots, \omega_{\tau-1}\right)=2 \tau-1 .
$$

Согласно леммам $3,4 \omega_{0}, \ldots, \omega_{\tau-1}$ алгебраически независимы над $\mathbb{C}(z)$, а по лемме 4 $\psi_{0}, \delta \psi_{0}, \ldots, \delta^{\tau-2} \psi_{0}$ алгебраически независимы над $\mathbb{C}(z)\left(\omega_{0}, \ldots, \omega_{\tau-1}\right)$, что доказывает равенство (34) и лемму в случае $c(u, v) \neq 0$.

б) Очевидно, что существует $\psi \in U_{\nu}$ такой, что $c(\psi, v) \neq 0$, так как в противном случае из (27) следует, что $v=0$.

Фиксируем базис $U_{\nu} \quad\left\{\psi_{0}=\psi / c(\psi, v), \psi_{1}=u, \psi_{2}, \ldots, \psi_{\tau-1}\right\}$ такой, что вьполнены все те же условия, что и в случае а).

Аналогично случаю а) получаем, что утверждение леммы равносильно условию (в обозначениях (33))

$$
\operatorname{deg} \operatorname{tr}_{\mathbb{C}(z)}\left(\psi_{1}, \delta \psi_{1}, \ldots, \delta^{\tau-2} \psi_{1}, \omega_{0}, \ldots, \omega_{\tau-1}\right)=2 \tau-1 .
$$

Равенство (35) непосредственно следует из лемм 3,4 . Лемма доказана.

Рассмотрим равенство (27) при $u=f_{\nu}(z), v=f_{\nu+g / 2}(z)$.

Положим (см. (22))

$$
\widetilde{c}= \begin{cases}0, & \text { если } X_{0}^{\prime} \neq 0, \\ (-1)^{s} X_{1}^{\prime 2} \cdots X_{s}^{\prime 2}, & \text { если } X_{0}^{\prime}=0 .\end{cases}
$$

Как и при вьводе равенства $(11)$, имеем $c(u, v)=0$ в случае $X_{0}^{\prime} \neq 0$. В случае $X_{0}^{\prime}=0$, подставляя в $(27)$ вместо $u, v$ соответствующие степенные ряды, получаем $c(u, v)=\gamma_{2}^{\prime} \cdots \gamma_{\tau}^{\prime}=\gamma_{2} \cdots \gamma_{\tau}=(-1)^{s} X_{1}^{\prime 2} \cdots X_{s}^{\prime 2}=\widetilde{c}$. Поэтому из (36) находим

$$
\begin{aligned}
\widetilde{c}= & f_{\nu}(z) \cdot\left(\delta+\gamma_{2}^{\prime}\right) \cdots\left(\delta+\gamma_{\tau}^{\prime}\right) f_{\nu+g / 2}(z)+f_{\nu+g / 2}(z) \cdot\left(\delta+\gamma_{1}\right) \cdots\left(\delta+\gamma_{\tau-1}\right) f_{\nu}(z) \\
& +\sum_{j=1}^{\tau-2}(-1)^{j}\left(\delta+\gamma_{1}\right) \cdots\left(\delta+\gamma_{j}\right) f_{\nu}(z) \cdot\left(\delta+\gamma_{j+2}^{\prime}\right) \cdots\left(\delta+\gamma_{\tau}^{\prime}\right) f_{\nu+g / 2}(z) \\
= & \sum_{k, r=0}^{\tau-1} \alpha_{k r}\left(\frac{1}{\tau} \delta\right)^{k} f_{\nu}(z)\left(\frac{1}{\tau} \delta\right)^{r} f_{\nu+g / 2}(z)
\end{aligned}
$$

где все $\alpha_{k r} \in \mathbb{Q}, \alpha_{k r}=0$, при $k+r \geqslant \tau$.

Осуществляя в равенстве $(37)$ замену $z \rightarrow(z / \tau)^{\tau}$ и учитывая $(19)$, получаем соотношение

$$
\sum_{k, r=0}^{\tau-1} \alpha_{k r} \delta^{k} \varphi_{\bar{\gamma}}\left(\xi^{\nu} z\right) \cdot \delta^{r} \varphi_{\bar{\gamma}}\left(\xi^{\nu+g / 2} z\right)=\widetilde{c} .
$$

Введем для $\nu=0, \ldots, g / 2-1$ обозначения

$$
\begin{gathered}
\bar{z}_{\nu}=\left(z_{0, \nu}, \ldots, z_{2 \tau-1, \nu}\right) \\
\bar{z}=\left(\bar{z}_{0}, \ldots, \bar{z}_{g / 2-1}\right)=\left(z_{i, \nu}, i=0, \ldots, 2 \tau-1, \nu=0, \ldots, \frac{g}{2}-1\right), \\
Q_{\nu}\left(\bar{z}_{\nu}\right)=\sum_{k, r=0}^{\tau-1} \alpha_{k r} z_{k, \nu} z_{\tau+r, \nu}-\widetilde{c}
\end{gathered}
$$


ЛЕмма 6. Идеал $J$, порожденный в кольие $\mathbb{C}(z)[\bar{z}]$ набором $g / 2$ многочленов $\left\{Q_{0}\left(\bar{z}_{0}\right), \ldots, Q_{g / 2-1}\left(\bar{z}_{g / 2-1}\right)\right\}$, является идеалом всех алгебраических связей над $\mathbb{C}(z)$ между функциями (17).

ДокаЗАтельство. Рассматривая равенство (31) при $v=f_{\nu+g} / 2$, находим, что $\widetilde{v}=$ $s\left((2-\tau) X_{0}^{\prime}\right) \cdot f_{\nu+g / 2}(z) \in \Phi_{\nu}, \nu=0, \ldots, g / 2-1$, поэтому из (37) и леммы 5 получаем, что для элементов поля $\Phi_{\nu}$ вьполнено

$$
\operatorname{deg} \operatorname{tr}_{\mathbb{C}(z)}\left(f_{\nu}(z), \ldots, \delta^{\tau-1} f_{\nu}(z), \widetilde{v}, \ldots, \delta^{\tau-1} \widetilde{v}\right)=2 \tau-1 .
$$

Ввиду (24) аналогично доказательству леммы 2 заключаем, что база трансцендентности поля $\Phi$ может быть получена объединением баз трансцендентности полей $\Phi_{0}, \ldots$, $\Phi_{g / 2-1} ;$ в частности, степень трансцендентности над $\mathbb{C}(z)$ набора функций $(17)$ равна $g(2 \tau-1) / 2=t-g / 2$.

Покажем, что все многочлены (39) неприводимы в кольце $\overline{\mathbb{C}(z)}\left[\bar{z}_{\nu}\right]$. Предположим противное. Тогда многочлен

$$
\widetilde{Q}_{\nu}=\sum_{k, r=0}^{\tau-1} \alpha_{k, r} z_{k, \nu} z_{\tau+r, \nu}
$$

приводим в кольце $\overline{\mathbb{C}(z)}\left[\bar{z}_{\nu}\right]$. Но многочлен $\widetilde{Q}_{\nu}$ однороден и имеет степень 1 по каждой группе переменных $\left(z_{0, \nu}, \ldots, z_{\tau-1, \nu}\right),\left(z_{\tau, \nu}, \ldots, z_{2 \tau-1, \nu}\right)$. Поэтому

$$
\widetilde{Q}_{\nu}=\left(h_{0} z_{0, \nu}+\cdots+h_{\tau-1} z_{\tau-1, \nu}\right)\left(\widetilde{h}_{0} z_{\tau, \nu}+\cdots+\widetilde{h}_{\tau-1} z_{2 \tau-1, \nu}\right),
$$

где все $h_{i}, \widetilde{h}_{i} \in \overline{\mathbb{C}(z)}$. Из $(37)$ и $(41)$ имеем

$$
\alpha_{0, \tau-1}=\tau^{\tau-1}=h_{0} \cdot \widetilde{h}_{\tau-1}, \quad \alpha_{\tau-1,0}=\tau^{\tau-1}=h_{\tau-1} \widetilde{h}_{0},
$$

т.е. $\widetilde{h}_{\tau-1} \neq 0, h_{\tau-1} \neq 0$. Тогда из (41) $\alpha_{\tau-1, \tau-1}=h_{\tau-1} \cdot \widetilde{h}_{\tau-1} \neq 0$, что невозможно ввиду условия $\alpha_{k, r}=0$ при $k+r \geqslant \tau$.

Итак, многочлены (39) неприводимы в кольце $\overline{\mathbb{C}(z)}\left[\bar{z}_{\nu}\right]$. В полной аналогии с доказательством леммы 2 заключаем, что идеал $J$ простой с общей точкой (17). Лемма доказана.

ДокаЗАТЕЛЬСтво теОРемЫ. Рассмотрим последовательно случаи 1), 2.1), 2.2) и 2.3).

1) Положим $y_{r}=\delta^{r} \varphi, r=0, \ldots, t-1, y_{t}=1$. Функции $y_{0}, \ldots, y_{t}$ составляют решение системы линейных однородных дифференциальных уравнений с коэффициентами из $\mathbb{C}(z)$, имеющей единственную особую точку $z=0$ (система (4) работы [2]). Пусть $\rho-$ простой идеал, порожденньй всеми однородными по $z_{0}, \ldots, z_{t}$ многочленами $R$ кольца $\mathbb{C}\left[z, z_{0}, \ldots, z_{t}\right]$ такими, что $R\left(z, y_{0}(z), \ldots, y_{t}(z)\right) \equiv 0 ; \rho_{\alpha}=\left\{R\left(\alpha, z_{0}, \ldots, z_{t}\right) \mid R \in \rho\right\}-$ однородньй идеал кольца $\mathbb{C}\left[z_{0}, \ldots, z_{t}\right]$. Согласно следствию 1 теоремы 1 работы [2] для доказательства теоремы достаточно показать, что

i) функции $y_{0}, \ldots, y_{t}$ линейно независимы над $\mathbb{C}(z)$;

ii) идеал $\rho_{\alpha}$ прост. 
Условие і) в этом и во всех остальных случаях выполняется (этот факт хорошо известен, см., например, [7, лемма 4.2] или [5, лемма 24]). Для проверки условия іi) ввиду замечания 1 достаточно ограничиться случаем (4). Условие іi) легко следует из леммы 1.

2.1) Утверждение теоремы в этом случае проще всего получить ввиду замечания 1 как непосредственное следствие теоремы Линдемана-Вейерштрасса.

2.2) Перенумеруем последовательно функции набора (17), т.е. если $k=s \tau+l$, где $0 \leqslant l \leqslant \tau-1,0 \leqslant s \leqslant g-1$, то $y_{k}=\delta^{l} \varphi \bar{\gamma}\left(\xi^{s} z\right), k=0, \ldots, t-1$. Согласно замечанию 1 достаточно установить справедливость условия іi) для набора функций (17). Обозначим для $s \in\{0, \ldots, g-1\}$ через $T_{s} \in \mathbb{C}\left[z_{s \tau}, \ldots, z_{s \tau+\tau-1}\right]$ многочлен, полученный подстановкой $z=\alpha$ и заменой $\bar{z}_{s} \rightarrow\left(z_{s \tau}, \ldots, z_{s \tau+\tau-1}\right)$ в многочлене $P_{s}\left(\bar{z}_{s}\right)$ или $\bar{P}_{s}\left(\bar{z}_{s}\right)$ (см. формулировку леммы 2). Ввиду леммы 2 простота идеала $\rho_{\alpha}$ является следствием простоты идеала $q$, порожденного многочленами $T_{0}, \ldots, T_{g-1}$ в кольце $\mathbb{C}\left[z_{0}, \ldots, z_{\tau-1}\right]$. Все многочлены $T_{s}$ неприводимы в кольце $\mathbb{C}\left[z_{s \tau}, \ldots, z_{s \tau+\tau-1}\right]$ (см. лемму 1 и равенства $(7),(9))$, поэтому главньй идеал $\left(T_{s}\right)$ при всех $s=0, \ldots, g-1$ определяет многообразие. Но тогда $[8$, с. 80] идеал $q$ прост, что и доказьвает теорему в случае 2.2$)$.

2.3) Этот случай полностью аналогичен предыдущему. Следует лишь воспользоваться леммой 6 и доказанной в этой лемме неприводимостью многочленов (40) в кольце $\mathbb{C}\left[\bar{z}_{\nu}\right]$.

Теорема полностью доказана.

В заключение авторы выражают благодарность проф. Ю.В. Нестеренко, обратившему их внимание на задачи, связанные с линейной независимостью значений $E$-функций, и проф. Д. Бертрану за полезные обсуждения, особенно в части работы, относящейся к дифференциальньм группам Галуа.

\section{СПИСОК ЦИТИРОВАННОЙ ЛИТЕРАТУРЫ}

[1] Салихов В.X. Критерий алгебраической независимости значений гипергеометрических $E$-функций (четный случай) // Матем. заметки. 1998. Т. 64. № 2. С. 273-284.

[2] Нестеренко Ю. В., Шидловский А. Б. О линейной независимости значений $E$-функций // Матем. сб. 1996. Т. 187. № 8. С. 93-108.

[3] Салихов В.Х. Критерий алгебраической независимости значений одного класса гипергеометрических $E$-функций // Матем. сб. 1990. Т. 181. № 2. С. 189-211.

[4] Салихов В. Х. Неприводимость гипергеометрических уравнений и алгебраическая независимости значений E-функций // Acta Arith. 1990. V. 53. № 5. P. 453-471.

[5] Салихов В. Х. Формальные решения линейных дифференциальных уравнений и их применение в теории трансцендентных чисел // Тр. ММО. 1988. Т. 51. С. 223-256.

[6] Katz N. M. Exponential Sums and Differential Equations. Ann. of Math. Stud. V. 124. Princeton: Princeton Univ. Press, 1990.

[7] Beukers F., Brownawell D., Heckman G. Siegel normality // Ann. of Math. 1988. V. 127. P. 279-308.

[8] Мерзляков Ю.И. Рациональные группы. М.: Наука, 1987.

Брянский институт транспортного машиностроения

Поступило

E-mail: lip@bgtu.brynsk.su

08.07.2001 